\title{
Piloting an Online New Member Orientation Program
}

\author{
Elizabeth V. Woodburn, WEMT
}

\section{POSTER PRESENTATION ABSTRACT}

Introduction: Within Illini EMS (IEMS) at the University of Illinois at Urbana-Champaign, entry-level membership as an EMS Aide requires only BLS certification. Aides are paired with a licensed EMT during operational events, but have historically arrived for their first volunteer shift knowing little more than basic CPR. Though hands-on training nights are held regularly, there is no requirement for Aides to attend. Program Development $\&$ Implementation: After identifying a need for formalized free training to prepare Aides to function within their team in a more comprehensive role, an online Orientation course was created and implemented. Using a combination of original and adapted videos followed by a quiz, this self-paced program teaches and assesses the basics of topics including bleeding control, organizational structure, team dynamics, and First Responder mental health in under an hour. Program Evaluation: After completing Orientation, Aides are required to complete a knowledge-based quiz and perception-based survey. Survey data confirms that, while not certifying them in any new skills, Orientation prepares them to integrate more effectively into the organization and understand the actions of their EMT teammates. $100 \%$ of respondents stated that Orientation improved their understanding of organizational structure, glove removal, and bleeding control. Discussion/Conclusion: As a collegiate EMS agency that sets its own membership requirements, this program allows us to forgo the formality and expense of relying on first aid certification for Aide training while streamlining our year-round onboarding of over 150 annual new members. Though hands-on training will continue to be an important part of onboarding, this tool will increase the quality of care that IEMS is able to provide without significantly raising the barrier to organizational entry. After this initial pilot of Aide-specific content, we hope to expand with separate modules to familiarize new EMTs with regional and organizational protocols. Future evaluations have been proposed to determine how well Aides retain this knowledge over time, and whether it translates to improved performance.

Author Affiliations: Illini Emergency Medical Services, Illini Fire Service Institute, University of Illinois at Urbana-Champaign, Urbana, IL, USA Address for Correspondence: Elizabeth V. Woodburn, WEMT. E-mail: woodbrn2@illinois.edu

Conflicts of Interest/Funding Sources: By the JCEMS Submission Declaration Form, all authors are required to disclose all potential conflicts of interest and funding sources. The author declared that they have no conflicts of interest. The author declared that they did not receive funding to conduct the program or research associated with this work.

Ethical Compliance: The authors attest that the research associated with this abstract was conducted in accordance with the JCEMS Ethics Guidelines.

Submission History: Received February 5, 2018; accepted for presentation and publication February 21, 2018.

Poster Presentation: This abstract was presented as a poster at the Academic Poster Session of the $25^{\text {th }}$ Annual Conference of the National Collegiate Emergency Medical Services Foundation; February 24, 2018; Philadelphia,
PA, USA. The authors received the Second Place Award for the Best Poster Presentation Competition. The poster is available for download on the JCEMS website.

Published Online: April 24, 2018

Published in Print: August 13, 2018 (Volume 1: Supplemental 1)

Reviewer Information: In accordance with JCEMS editorial policy, poster presentation abstracts undergo double-blind peer-review by at least two reviewers (JCEMS Editorial Board members and/or independent reviewers) prior to acceptance for presentation and publication. JCEMS thanks the anonymous reviewers who contributed to the review of this work.

Copyright: (C) 2018 Woodburn. This is an open access abstract distributed under the terms of the Creative Commons Attribution 4.0 International (CC BY 4.0) License, which permits unrestricted use, distribution, and reproduction in any medium, provided the original author and source are credited. The full license is available at: https://creativecommons.org/licenses/by/4.0/

Electronic Link: https://doi.org/10.30542/JCEMS.2018.01.S1.02 